\title{
Four new HgMn stars: HD 18104, HD 30085, HD 32867, and HD 53588 (Research Note)
}

\author{
R. Monier ${ }^{1,2}$, M. Gebran ${ }^{3}$, and F. Royer ${ }^{4}$ \\ ${ }^{1}$ LESIA, UMR 8109, Observatoire de Paris, Place J. Janssen, 92195 Meudon, France \\ 2 Laboratoire Lagange, Université de Nice Sophia, Parc Valrose, 06100 Nice, France \\ e-mail: Richard.Monier@unice.fr \\ 3 Department of Physics and Astronomy, Notre Dame University-Louaize, PO Box 72, Zouk Mikael, Lebanon \\ ${ }^{4}$ GEPI, Observatoire de Paris, Place J. Janssen, 92195 Meudon, France
}

Received 16 March 2015 / Accepted 10 April 2015

\section{ABSTRACT}

\begin{abstract}
Context. We have discovered four new HgMn stars while monitoring a sample of apparently slowly rotating superficially normal bright late-B and early-A stars in the northern hemisphere.

Aims. Important classification lines of $\mathrm{Hg}$ II and Mn II are found as conspicuous features in the high resolution SOPHIE spectra of these stars $(R=75000)$.

Methods. Several lines of $\mathrm{Hg}$ II, Mn II and Fe II were synthesized using model atmospheres and the spectrum synthesis code SYNSPEC48, including hyperfine structure of various isotopes when relevant. These synthetic spectra were compared to highresolution observations of these stars that have a high signal-to-noise ratio to derive abundances of these key elements.

Results. The four stars are found to have distinct enhancements of $\mathrm{Hg}$ and $\mathrm{Mn}$, which shows that they are not superficially normal B and A stars, but are new HgMn stars and need to be reclassified as such.
\end{abstract}

Key words. stars: abundances - stars: atmospheres - stars: chemically peculiar - stars: early-type

\section{Introduction}

We have recently undertaken a spectroscopic survey of all apparently slowly rotating bright early-A (A0-A1V) and late-B stars (B8-B9V) observable from the northern hemisphere. The incentive is to search for rapid rotators seen pole-on or new chemically peculiar B and A stars that have thus far remained unnoticed. The abundance results for the A0-A1V sample have been published in Royer et al. (2014). The selection criteria were a declination higher than $-15^{\circ}$, spectral class A0 or A1 and luminosity class $\mathrm{V}$ and $\mathrm{IV}$, and magnitudes $V$ brighter than 6.65 . The B8-9 sample employs the same criteria, except for the $V$ magnitude brighter than 7.85 because these B stars are intrinsically brighter in the $V$ band where SOPHIE reaches its highest efficiency. Most of the stars of that B8-9 sample (40 stars) have just recently been observed in December 2014. A careful abundance analysis of the spectra with a high resolution and high signalto-noise ratio $(\mathrm{S} / \mathrm{N})$ of the A stars sample has allowed sorting out the sample of $47 \mathrm{~A}$ stars into 17 chemically normal stars (whose abundances do not depart by more than \pm 0.20 dex from solar values), 12 spectroscopic binaries, and 13 chemically peculiar stars (CPs), five of which are new CP stars. The status of these new CP stars still needs to be fully specified by spectropolarimetric observations to address their magnetic nature or by exploring new spectral ranges that we had not explored in this first study. Indeed, the abundance analysis of the A stars sample in Royer et al. (2014) only relied on four spectral regions: 4150-4300 $, 4400-4790 \AA, 4920-5850 \AA$, and 6000-6275 , avoiding Balmer lines and atmospheric telluric lines.
We have now started to examine the A0-A1V and B9$\mathrm{B} 8 \mathrm{~V}$ samples in the region of the red wing of the $\mathrm{H} \epsilon$ line to search for the $\mathrm{Hg}$ II $3983.93 \AA$ line and also the spectral range $4125-4145 \AA$, which harbors the Mn II line at $4136.92 \AA$ next to the Si II doublet (multiplet 2). The Hg II $3983.93 \AA$ A line and the Mn II line at $4136.92 \AA$ lines with appreciable strengths are the signatures of an HgMn star (Jaschek \& Jaschek 1995). Note that, a priori, only one of these two elements ( $\mathrm{Mn}$ or $\mathrm{Hg}$ ) is sufficient to identify a new HgMn star because both elements may be distributed in different spots and may not be simultaneously visible to the observer at a given time (see Hubrig et al. 2012 for a complete review). In our A and B stars samples, we found that four stars unambiguously show the $\mathrm{Hg}$ II line at $3984 \AA$ and the Mn II line at $4136.92 \AA$ (and several other strong Mn II lines). These are HD 18104 (B9V), HD 30085 (A0IV), HD 32867 (B8V), and HD 53588 (B9V). A bibliographic query of the CDS for each of these stars revealed very few publications (about ten references each). HD 30085 was ascribed a spectral type A0IV in the survey of Cowley et al. (1969) of bright A stars (observed with prismatic dispersion of $125 \AA^{-1} \mathrm{~mm}^{-1}$ around $\mathrm{H} \gamma$ ), and the authors did not mention any peculiarity in the spectrum. The other three B stars do not appear in Cowley's classification (1972) of the bright B8 stars. The purpose of this research note is to report on the detection of the $\mathrm{Hg}$ II $3983.93 \AA$ line and strong Mn II lines as well in these four stars that are currently classified as "normal". We have also determined estimates of the iron, manganese, and mercury abundances using spectrum synthesis to quantify the enhancements of these elements in these stars. 
Table 1. Observation log.

\begin{tabular}{cccccc}
\hline \hline Star ID & $\begin{array}{c}\text { Spectral } \\
\text { type }\end{array}$ & $V$ & $\begin{array}{c}\text { Observation } \\
\text { date }\end{array}$ & $\begin{array}{c}\text { Exposure } \\
\text { time (s) }\end{array}$ & $S / N$ \\
\hline HD 18104 & B9 & 6.85 & $2014-12-16$ & 800 & 136 \\
HD 30085 & A0IV & 6.35 & $2013-12-11$ & 1200 & 269 \\
& & & $2012-13-03$ & 800 & 216 \\
HD 32867 & B8V & 7.48 & $2014-12-16$ & 2100 & 170 \\
HD 53588 & B9V & 7.20 & $2014-12-17$ & 1350 & 158 \\
\hline
\end{tabular}

\section{Observations and data reduction}

The four stars have been observed at Observatoire de Haute Provence using the high-resolution $(R=75000)$ mode of the SOPHIE echelle spectrograph (Perruchot et al. 2008) at three different epochs: February 2012, December 2013, and December 2014. The three late-B stars have been observed only once in December 2014. The A0IV star HD 30085 was observed twice, in February 2012 and December 2013. The coaddition of the two exposures for HD 30085 yields a coadded spectrum whose $\mathrm{S} / \mathrm{N}$ is about 300 . The three late B stars observed in December 2014 have lower $\mathrm{S} / \mathrm{N}$ ratios ranging from 136 to 170. The observations log is displayed in Table 1 . The data were automatically reduced to produce 1D extracted and wavelength calibrated échelle orders. Each reduced order was normalised separately using a Chebychev polynomial fit with sigma clipping, rejecting points above or below $1 \sigma$ of the local continuum. Normalised orders were merged, corrected by the blaze function, and resampled into a constant wavelength step of about $0.02 \AA$ (see Royer et al. 2014, for more details).

Radial velocities were derived from cross-correlation techniques, avoiding the Balmer lines and the atmospheric telluric lines. The normalised spectra were cross-correlated with a synthetic template extracted from the POLLUX database ${ }^{1}$ (Palacios et al. 2010) corresponding to the parameters $T_{\text {eff }}=11000 \mathrm{~K}$, $\log g=4$, and a solar metallicity. A parabolic fit of the upper part of the resulting cross-correlation function yielded the Doppler shifts, which were then used to shift spectra to rest wavelengths. The radial velocity of HD 30085 is found to be the same within its accuracy at the two different epochs of observations: $V_{\text {rad }}=8.27 \pm 0.20 \mathrm{~km} \mathrm{~s}^{-1}$. We only searched for line profile variability in the lines of $\mathrm{Hg}$ II and $\mathrm{Mn}$ II. The individual spectra of HD 30085 were coadded and the difference of each spectrum with the mean spectrum was computed. This difference was then ratioed to the estimated noise level $\sigma$ of the local continuum. We considered real variations only if the absolute value of the difference was found to be higher than $3 \sigma$. We failed to find any real line profile variations or radial velocity variations above three $\sigma$ of the noise level for HD 30085.

\section{Hg II and Mn II lines in the four stars}

Two spectral regions were used to establish the $\mathrm{HgMn}$ nature of the four stars. First, the red wing of $\mathrm{H} \epsilon$, which lies in order 3, harbours the $\mathrm{Hg}$ II $\lambda 3983.93 \AA$ line and several Zr II and Y II lines that are expected to be strengthened in HgMn stars. Jaschek \& Jaschek (1995) emphasised that the Hg II $3983.93 \AA$ line is absent from the spectra of normal late-B stars. Second, the region from $4125 \AA$ to $4145 \AA$ (order 6) contains the Mn II line at $4136.92 \AA$ redwards of the Si II doublet (multiplet 2). In a HgMn star, the Mn II line at $4136.92 \AA$ should be strong, but

\footnotetext{
1 http://pollux.graal.univ-montp2 .fr
}

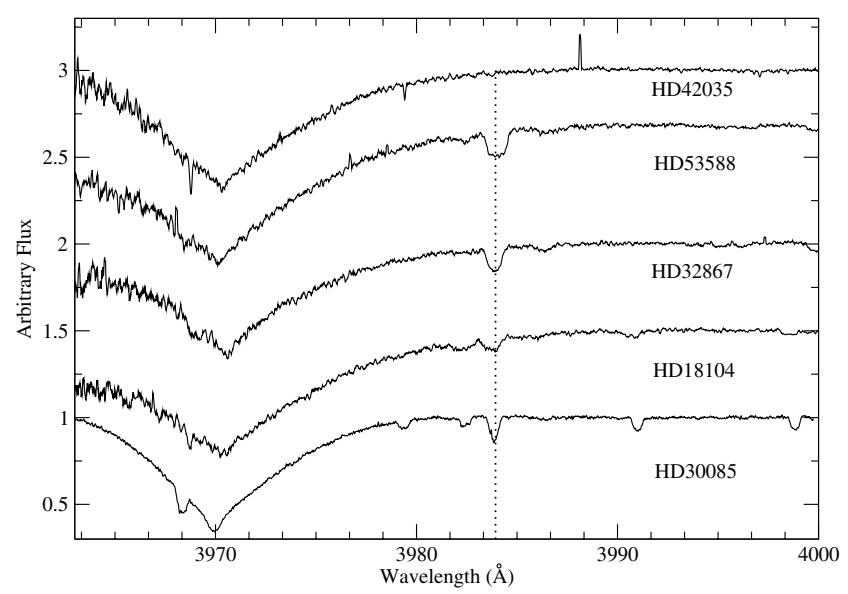

Fig. 1. Detection of the $\mathrm{Hg}$ II line at $3984 \AA$; its rest wavelength location is depicted as a vertical line in the spectra of the four stars. The spectra have been offset from each other for clarity. The $\mathrm{Hg}$ II line is absent from the spectrum of the comparison star HD 42035.

it should be absent from any comparison normal late B-type star. Furthermore, the lines of Mn II at $4206.37 \AA$ and $4252.96 \AA$ should also be enhanced in the spectra of HgMn stars (Gray \& Corbally 2009).

Figure 1 displays order 3 for the four stars and a comparison star, HD 42035 (a normal B9 V star of our sample) and the location as a vertical line of the rest wavelength of the Hg II $3983.93 \AA$ line (Multiplet 2), Fig. 2 displays the Mn II line at $4136.92 \AA$ longwards of the Si II doublet (Multiplet 2) at 4128.07 $\AA$ and $4130.88 \AA$. The Hg II $3983.93 \AA$ line and the Mn II $4136.92 \AA$ are clearly present in the four spectra of the four stars and absent in the comparison star HD 42035. The equivalent width of the $\mathrm{Hg}$ II $3983.84 \AA$ line ranges from $64 \mathrm{~m} \AA$ to $132 \mathrm{~m} \AA$, which is well in the range quoted for HgMn stars (from $50 \mathrm{~m} \AA$ up to $300 \mathrm{~m} \AA$ typically in Jaschek \& Jaschek 1995). The equivalent width (EW) of the Mn II line at $4136.92 \AA$ ranges from $47.4 \mathrm{~m} \AA$ up to $93.6 \mathrm{~m} \AA$ in the four stars, but this Mn II line is absent from the spectrum of the comparison star HD 42035. The Mn II lines at $4206.37 \AA$ and $4252.96 \AA$ are also prominent features in the spectra of all stars. The EWs of $4206.37 \AA$ ranges from $66.7 \mathrm{~m} \AA$ to $117.4 \mathrm{~m} \AA$, in agreement with Kodaira \& Takada (1978), who found a mean EW of about $100 \mathrm{~m} \AA$ for the $4206.37 \AA$ line for a group of HgMn stars. The EW of $4252.96 \AA$ ranges from $75 \mathrm{~m} \AA$ up to $122.4 \mathrm{~m} \AA$. The comparison of the spectra of the four stars to that of the normal stars in these key regions leads us to propose that these stars are new HgMn stars and should be reclassified as such.

\section{Abundance determinations}

\subsection{Fundamental parameter determinations}

For the three B stars, we adopted the effective temperatures and surface gravities derived by Huang et al. (2010) from fitting the $\mathrm{H} \gamma$ profiles. Two B stars, HD 18104 and HD 53588, do not have Strömgren photometry, which precludes using the UVBYBETA procedure developed by Napiwotzky (1993) to derive their fundamental parameters. We therefore used the effective temperature and surface gravity derived in Huang et al. (2010) by fitting the $\mathrm{H} \gamma$ profile for the three stars for consistency. For HD 30085 , the effective temperature and surface gravity were derived from Strömgren photometry in Royer et al. (2014), and a spectrum 
R. Monier et al.: Four new HgMn stars $(R N)$

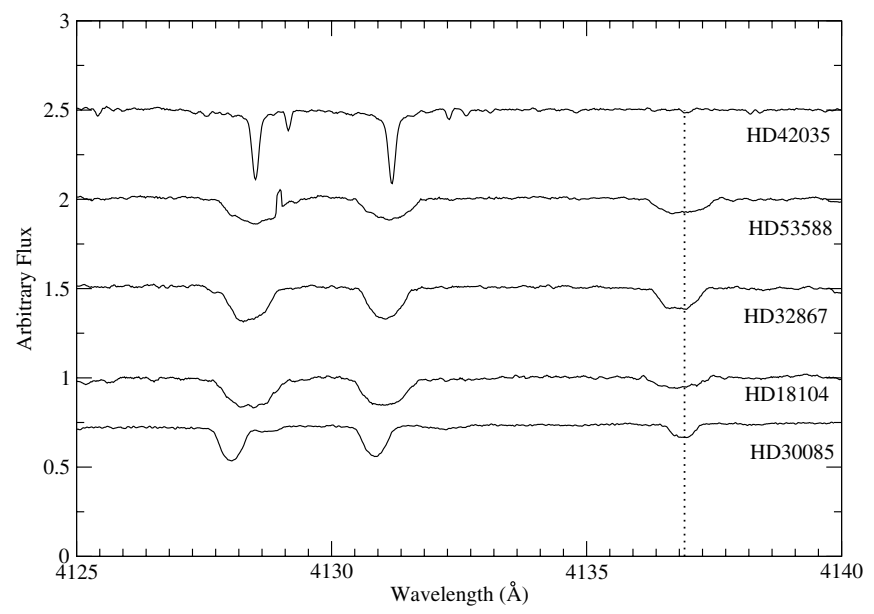

Fig. 2. Detection of the Mn line at $4136.92 \AA$; its rest wavelength location is depicted as a vertical line in the spectra of the four stars. The spectra have been offset from each other for clarity. The Mn II line is absent from the spectrum of the comparison star HD 42035.

Table 2. Stellar fundamental parameters.

\begin{tabular}{ccccc}
\hline \hline Star ID & $T_{\text {eff }}$ & $\log g$ & $\begin{array}{c}v \sin i \\
\left(\mathrm{~km} \mathrm{~s}^{-1}\right)\end{array}$ & $\begin{array}{c}V_{\text {rad }} \\
\left(\mathrm{km} \mathrm{s}^{-1}\right)\end{array}$ \\
\hline HD 18104 & 11074 & 3.67 & 46.0 & 12.07 \\
HD 30085 & 11300 & 3.95 & 23.0 & 8.20 \\
HD 32867 & 13149 & 3.86 & 37.0 & 13.97 \\
HD 53588 & 12351 & 3.88 & 48.0 & 12.03 \\
\hline
\end{tabular}

synthesis of its $\mathrm{H} \gamma$ profile was run to confirm these parameters. The adopted effective temperatures, surface gravities, and projected equatorial velocities are displayed in Table 2.

\subsection{Model atmospheres and spectrum synthesis calculations}

Plane-parallel model atmospheres assuming radiative equilibrium and hydrostatic equilibrium were computed using the ATLAS9 code (Kurucz 1992). The linelist was built from Kurucz (1992) gfhyperall.dat ${ }^{2}$, which includes hyperfine splitting levels. A grid of synthetic spectra was computed with SYNSPEC48 (Hubeny \& Lanz 1992) to model the Hg II, Mn II, Fe II, and Si II lines. Computations were iterated by varying the unknown abundance until minimisation of the chi-square between the observed and synthetic spectrum was achieved. The microturbulent velocities were assumed to be 0 or $0.5 \mathrm{~km} \mathrm{~s}^{-1}$, in agreement with most analyses of $\mathrm{HgMn}$ stars whose atmospheres are thought to be very quiet.

\subsection{Derived iron, manganese, and mercury abundances}

The iron abundances were derived by using several Fe II lines of multiplets 37,38 , and 186 in the range 4500-4600 $\AA$, whose atomic parameters are critically assessed in NIST ${ }^{3}$ (these are C+ and D quality lines). These lines are widely spaced, and the continuum is fairly easy to trace in this spectral region. Their synthesis always yields consistent iron abundances from the various transitions with very little dispersion. The iron abundance is

\footnotetext{
2 http://kurucz.harvard.edu

3 http://www.nist.gov
}

Table 3. Abundance determinations.

\begin{tabular}{cccc}
\hline \hline Star ID & $\begin{array}{c}\text { Chemical } \\
\text { element }\end{array}$ & $\begin{array}{c}\text { Laboratory } \\
\text { wavelength }(\AA)\end{array}$ & {$\left[\frac{N_{\text {elem }}}{N_{\text {solar }}}\right]$} \\
$\odot$
\end{tabular}

probably the most accurately determined of the three abundances derived here. We find that the four stars show only mild enhancement in iron, about 2 to 2.5 solar. The Fe II, Mn II, and $\mathrm{Hg}$ II abundances and their estimated uncertainties for the four selected stars are collected in Table 3 (the determination of the uncertainties is discussed in Royer et al. 2014).

The manganese abundances were derived from two $\mathrm{C}+$ quality lines, $\lambda 4206.368 \AA$ and $4259.191 \AA$, whose hyperfine structure is published in Holt et al. (1999). The individual transitions were added to our intitial lineslist using the wavelengths, oscillator strengths, and angular momenta from Table 1 in Holt et al. (1999). We did not use the other two Mn II lines at $4326.644 \AA$ and $4348.396 \AA$ analysed by Holt et al. (1999) because they fall in the blue and red wings of the $\mathrm{H} \gamma$ line where the continuum is more difficult to locate. After the hyperfine structure of 4206.368 $\AA$ and $4259.191 \AA$ were properly taken into account, these two Mn II lines yielded very consistent abundances that were significantly lower than when the hyperfine structure was ignored (up to a factor of ten lower). Manganese enrichments range from 40 up to 350 solar and appear to slowly increase with effective temperature, the two coolest stars (HD 18104 and HD 30085) around $11000 \mathrm{~K}$ having the lowest manganese enrichment (40-50 $\odot$ ), while the hottest star, HD 32867, is the most enriched. This agrees with the correlation of manganese abundances with the effective temperature reported by Smith \& Dworetsky (1993) using ultraviolet lines of Mn II for a large number of $\mathrm{HgMn}$ stars.

The mercury abundances were derived from the Hg II $3983.93 \AA$ line in NIST (multiplet 2) but including the hyperfine structure of the various isotopes as provided in Dolk et al. (2003). We used the wavelengths and oscillator strengths for each transition as presented in their Table 2 for the case of a terrestrial isotopic mixture. The other line of $\mathrm{Hg}$ II that might be present in the spectra of HgMn stars is that of multiplet 4 at $6149.48 \AA$, but it appears to be blended in all four stars with the Fe II line at $6149.25 \AA$ so that we did not use this line for abundance analysis. Dolk et al. (2003) assumed a terrestrial isotope mixture for mercury, which may not occur in the $\mathrm{HgMn}$ stars we investigate. Note that the $3983.93 \AA$ line is blended with two lines, CrI $3983.897 \AA$ and Fe I $3983.960 \AA$, which contribute no absorption at the temperatures of the late-B stars we study here (this is easily verified by removing the $\mathrm{Hg}$ II hyperfine transitions from the line synthesis and by checking that no absorption is computed for the appropriate iron abundance for any of these stars). The 




Fig. 3. Comparison of the observed Hg II $3983.93 \AA$ line profile of HD 32867 to a synthetic profile computed for an overabundance of 130000 solar. The vertical line depicts the location of the rest wavelength of the $\mathrm{Hg}$ II line.

$\mathrm{Hg}$ overabundances range from 32000 to 300000 solar, where again the coolest stars tend to have lower $\mathrm{Hg}$ enhancement than the hotter stars. The uncertainties on these abundances are believed to be of the order of $\pm 2000 \odot$ to $\pm 27000 \odot$. The synthetic spectrum reproducing the best the $\mathrm{Hg}$ II profile for HD 32867 for a 130000 solar overabundance of $\mathrm{Hg}$ is compared to the observed profile rectified to the red wing of $\mathrm{H} \epsilon$ in Fig. 3. Only the mean spectrum of HD 30085 is of sufficient quality to examine the structure of the line core. In the two observations of this star, the core of the $3983.93 \AA$ line appears to be fairly flat and extending from $3893.90 \pm 0.02 \AA$ to $3984.07 \pm 0.02 \AA$, which roughly corresponds to the positions of the transitions of the heaviest isotopes ${ }^{200} \mathrm{Hg}$ and ${ }^{204} \mathrm{Hg}$. The accuracy of the wavelength scale is achieved by using four control lines on each side of the $\mathrm{Hg}$ II line: shortwards of the $\mathrm{Zr}$ II line at 3982.025 $\AA$, the Y II line (M 6) at $3982.59 \AA$, and longwards of the $\mathrm{Zr}$ II lines at $3984.718 \AA$ and $3991.15 \AA$ : after correcting for the radial velocity of HD 30085, the centres of these lines are found at their expected laboratory locations to within $\pm 0.02 \AA$. These two isotopes appear thus to contribute most of the absorption in HD 30085, as is the case in the majority of the coolest
HgMn stars (White et al. 1976), whereas they only contribute a small fraction of the terrestrial mixture.

\section{Conclusions}

We have found four new $\mathrm{HgMn}$ stars from the inspection of the $\mathrm{H} \epsilon$ wing and a few Mn II lines. We provide for the first time estimates of the overabundances of $\mathrm{Fe}, \mathrm{Mn}$, and $\mathrm{Hg}$ in these stars, which were until now considered as normal. They clearly must be reclassified as $\mathrm{HgMn}$ stars. The detection of these new $\mathrm{HgMn}$ stars is quite important because they only represent about $8 \%$ for the coolest B-type stars around B9 and B8 (Wolff \& Preston 1978). We expect to find other new HgMn stars by extending our survey to the late-B stars of the southern hemisphere. Each of these stars will be the subject of a detailed abundance analysis, which we plan to publish in the near future.

Acknowledgements. We thank the referee, S. Hubrig, whose comments resulted in many improvements. We also thank the OHP night assistants for their helpful support during the three observing runs.

\section{References}

Cowley, A. 1972, AJ, 77, 750

Cowley, A., Cowley, C., Jaschek, M., \& Jaschek, C. 1969, AJ, 74, 375

Dolk, L., Wahlgren, G. M., \& Hubrig, S. 2003, A\&A, 402, 299

Gray, R., \& Corbally, J. C. 2009, Stellar spectral classification (Princeton University Press), 133

Huang, W., Gies, D. R., \& Mc Swain, M. 2010, ApJ, 722, 605

Hubeny, I., \& Lanz, T. 1992, A\&A, 262, 501

Hubrig, S., González, J. F., Ilyin, I., et al. 2012, A\&A, 547, A90

Holt, R., A., Scholl, T. J., \& Rosner, S. D. 1999, MNRAS, 306, 107

Jaschek, C., \& Jaschek, M., 1995, The behavior of chemical elements in stars (Cambridge University Press), 111

Kodaira, K., \& Takada, M. 1978, Ann Tokyo Obs, second Ser., 17, 79

Kurucz, R. L. 1992, Rev. Mex. Astron. Astrofis., 23, 45

Napiwotzki, R., Schoenberner, D., \& Wenske, V. 1993, A\&A, 268, 653

Palacios, A., Gebran, M., Josselin, E., et al. 2010, A\&A, 516, A13

Perruchot, S., Kohler, D., Bouchy, F., et al. 2008, in Ground-based and Airborne Instrumentation for Astronomie II, eds. J. S. McLean, \& M. M. Casali, Proc. SPIE, 7014

Royer, F., Gebran, M., Monier, R., et al. 2014, A\&A, 562, A84

Smith, K. C., \& Dworetsky, M. M. 1993, A\&A, 274, 335

White, R. E., Vaughan, A. H., Preston, G. W., \& Swings, J. P. 1976, ApJ, 204, 131

Wolff, S. C., \& Preston, G. W. 1978, ApJS, 37, 371 